\title{
Kondo-excitons and Auger processes in self-assembled quantum dots
}

\author{
A. O. Govorov ${ }^{1,2}$, K. Karrai ${ }^{3}$, R. J. Warburton ${ }^{4}$, and A. V. Kalameitsev ${ }^{2}$ \\ ${ }^{1}$ Department of Physics and Astronomy, Ohio University, Athens, Ohio 45701, USA \\ ${ }^{2}$ Institute of Semiconductor Physics, 630090 Novosibirsk, Russia \\ ${ }^{3}$ Center for NanoScience and Sektion Physik, Ludwig-Maximilians-Universität, 80539 \\ München, Germany \\ ${ }^{4}$ Department of Physics, Heriot-Watt University, Edinburgh EH14 4AS, UK
}

\begin{abstract}
We describe theoretically novel excitons in self-assembled quantum dots interacting with a two-dimensional (2D) electron gas in the wetting layer. In the presence of the Fermi sea, the optical lines become strongly voltage-dependent. If the electron spin is nonzero, the width of optical lines is given by $k_{B} T_{K}$, where $T_{K}$ is Kondo temperature. If the spin is zero, the exciton couples with the continuum due to Auger-like processes. This leads to anticrossings in a magnetic field. Such states can be called Kondo-Anderson excitons. Some of the described phenomena are observed in recent experiments.
\end{abstract}

\section{INTRODUCTION}

Many-body phenomena and excitons in quantum dots (QDs) attract presently much interest. The number of electrons in QDs is voltage tunable. This makes it possible to study single electron effects. One example of many-body phenomena is the Kondo effect induced by the non-zero spin. So far, the Kondo effect was studied mostly in relation to transport properties [1]. In optics, Kondo-type effects were discussed with respect to nonlinear shakeup processes in nanostructures [2].

Here we study theoretically novel exciton states in self-assembled QDs coupled with delocalized electron states. If the electron spin of the QD is non-zero, the resulting states can be called Kondo-excitons. The Kondo temperature of these excitons is given by the small dimensions of QDs and can be as high as $10 \mathrm{~K}$. The Kondo effect manifests itself as peculiar, temperature-dependent optical lines. If the exciton spin is zero, a hybridization of the final state can occur due to Auger-like processes. Another effect is the transition between different exciton ground states on increasing the Fermi energy. In the transition regime, the optical lines become strongly voltage-dependent. Some of above-mentioned effects have been observed in optical spectra of single InAs QDs [3].

Charged excitons $X^{n-}$, observed in experiments [3, 4, 5], contain $n+1$ electrons and one hole. In the exciton, the hole is optically excited; the electrons are supplied by tunneling from the back contact and by optical excitation (fig.1).

The voltage applied between the top and back contact, $U_{\text {gate }}$, makes it possible to control the number of electrons in a QD. If $U_{\text {gate }}$ is sufficiently high, electrons fill the wetting layer, a 2D quantum well. The Fermi energy of a 2D gas in the wetting layer is found from the conditions of equilibrium, $\epsilon_{F}=\left(a_{0}^{*} / 4 d\right) \Delta U_{\text {gate }}$, where $a_{0}^{*}$ is the Bohr radius and $\Delta U_{\text {gate }}$ is the voltage measured from the point at which the wetting layer starts to load [6]. In our model, an asymmetric QD has two bound, non-degenerate states with energies $E_{s}^{e}$ and $E_{p}^{e}$. The 
a)

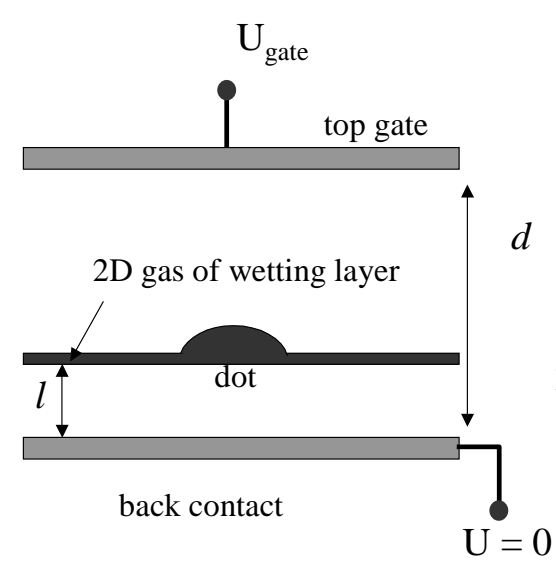

b)

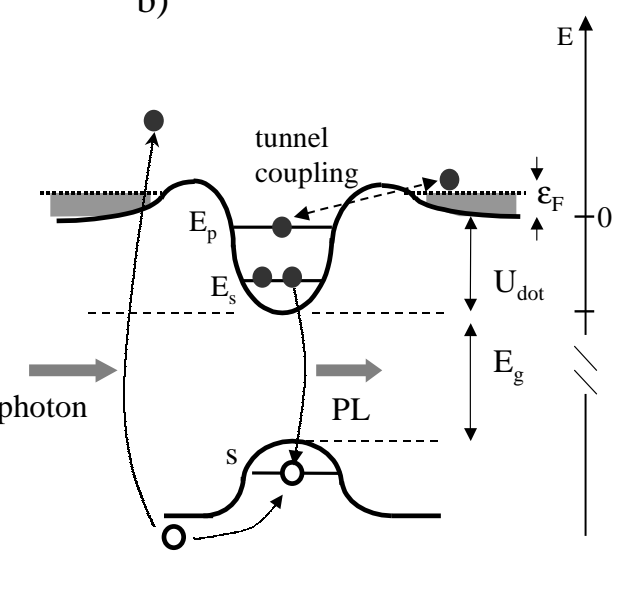

FIG. 1: (a) Sketch of the system with quantum dots embedded between a top gate and back contact. By application of a voltage, one can load single electrons in the QD and change the Fermi energy in the wetting layer; $l \ll d$. (b) The band diagram of a quantum dot and wetting layer.

indexes $s$ and $p$ originate from electronic shells in a symmetric QD. The system is described by the Anderson Hamiltonian,

$$
\hat{H}=\hat{H}_{s p}+\hat{H}_{\text {Coul }}^{\text {dot }}+\hat{H}_{\text {tun }}, \quad \hat{H}_{\text {tun }}=\sum_{\sigma} V_{k}\left[c_{k, \sigma}^{+} a_{p, \sigma}+a_{p, \sigma}^{+} c_{k, \sigma}\right],
$$

where $\hat{H}_{s p}$ is the single-particle energy; $\hat{H}_{C o u l}^{\text {dot }}$ is the operator for intra-dot Coulomb interactions, and $\hat{H}_{t u n}$ is the operator for a tunnel hybridization between the QD and Fermi sea; $c_{k, \sigma}$ is the operator of a delocalized electron with a momentum $k$ and spin $\sigma ; \epsilon_{k}$ denotes the kinetic energy of delocalized electrons; $a_{p, \sigma}^{+}$is related to the $p$-state of QD. Here we assume that the tunnel coupling occurs only between the upper $p$-state of the QD and the Fermi sea (fig. 1). In our simplified approach, the $p$-electron moves in a potential having a barrier at the edges of the QD. This barrier is formed by the short-range QD potential and Coulomb repulsion from the $s$-electrons.

We assume that $\Delta E>U_{\text {Coul }}>E_{\text {hyb }}$, where $\Delta E=E_{p}^{e}-E_{s}^{e}$ and $U_{\text {Coul }}$ is the intra-dot Coulomb energy, and $E_{h y b}$ is the energy due to the hybridization between the QD and the Fermi sea. Thus, the Coulomb interaction in a QD can be included with perturbation theory.

The most intense lines observed in the PL spectra of QDs [4, 5] are related to the transitions between the $s$-states in the conduction and valence bands. The PL spectrum is given by a Green function:

$$
I(\omega)=\operatorname{Re} \int_{0}^{\infty} d t e^{-i \omega t}<\hat{V}_{o p t}^{+}(t) \hat{V}_{o p t}(0)>, \quad \hat{V}_{o p t}=V_{o p t}\left(b_{s,-\frac{3}{2}} a_{s, \uparrow}+b_{s, \frac{3}{2}} a_{s, \downarrow}+c . c .\right),
$$

where $\omega$ is the frequency of the emitted photon and $\hat{V}_{\text {opt }}$ is the electron-photon interaction, involving the $s$-states of electrons and heavy holes $(J=3 / 2)$. An averaging in eq. 2 involves 


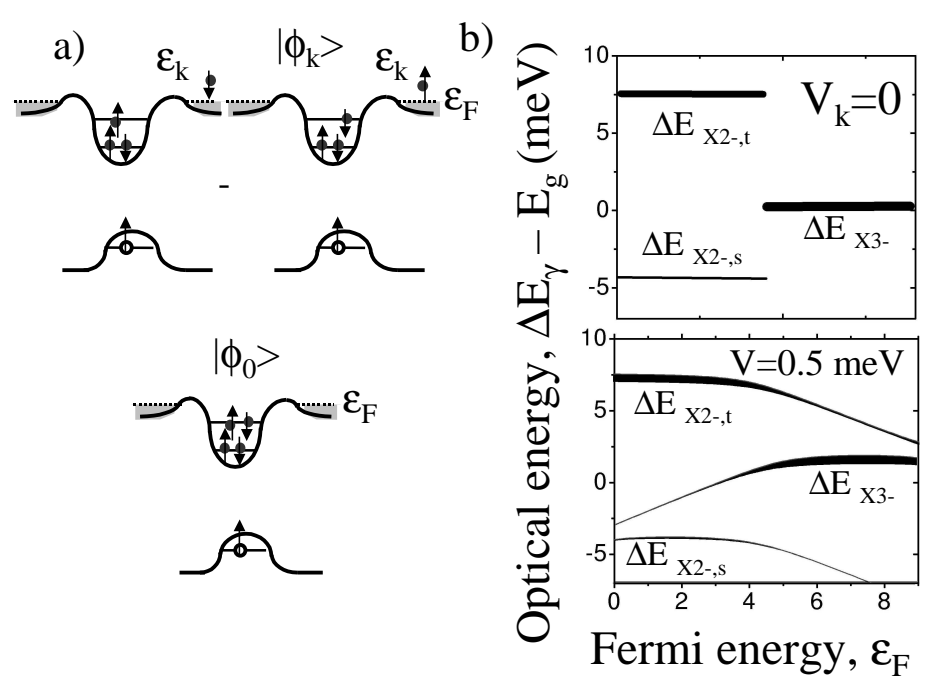

FIG. 2: (a) Contributions to the wave functions of excitons $X^{2-}$ and $X^{3-}$. (b) The PL spectrum as a function of the Fermi energy with and without hybridization (upper and lower panels). The width of the lines is proportional to the intensity. $U_{d o t}=64 \mathrm{meV}$.

all initial states at a finite temperature $T$. First we consider the limit $V_{k} \rightarrow 0$ and $T \rightarrow 0$. At small Fermi energies $\epsilon_{F}$, the ground state of the system is the exciton $X^{2-}$ (fig. 2a, upper part). With increasing $\epsilon_{F}$, the exciton changes its charge and the ground state becomes $X^{3-}$ (fig. 2a, lower part). The transition Fermi energy $\epsilon_{1 \rightarrow 2}$ is determined by the equation,

$$
E_{X 2-}+\epsilon_{1 \rightarrow 2}=E_{X 3-},
$$

where $E_{X 2-}$ and $E_{X 3-}$ are intra-dot exciton energies. In fig. 2b we show the PL spectrum as a function of $\epsilon_{F}$ in the absence of hybridization $\left(V_{k}=0\right)$. At $\epsilon_{F}=\epsilon_{1 \rightarrow 2}$, the spectrum changes abruptly because of the ground-state transition. In the regime $\epsilon_{F}<\epsilon_{1 \rightarrow 2}$, the spectrum has two lines, $\Delta E_{X 2-, s}$ and $\Delta E_{X 2-, t}$, related to the singlet and triplet final states [4]. The splitting between these lines is due to the exchange interaction in the exciton. To calculate the optical energies, we use a model of an anisotropic harmonic oscillator. The oscillator energies are taken as follows: $\hbar \Omega_{x}^{e}=20 \mathrm{meV}, \hbar \Omega_{y}^{e}=25 \mathrm{meV}$, and $\Omega_{x, y}^{h}=\Omega_{x, y}^{e} / 2$, where the upper indexes denote electrons (holes).

\section{KONDO EXCITON $X^{2-}$ WITH $S_{d o t}=1 / 2$}

Now we focus on the interaction between the QD and 2D electrons, assuming $V_{k} \neq 0$ and $T=0$. In the exciton $X^{2-}$, the intra-dot electron spin is $S_{d o t}=1 / 2$. So, the exciton wave function is mixed with the Fermi sea, leading to a Kondo state with $S_{e}=0$, where $S_{e}$ is the total electron spin (fig. 2a). A trial function for the Kondo exciton can be written as [7]:

$$
\begin{array}{r}
\Psi_{\text {initial }}=\left[A_{0}\left|\phi_{0}>+\sum_{k>k_{F}} A_{k}\right| \phi_{k}>\right] * \mid \uparrow, 0>_{h}, \\
\left|\phi_{0}>=\right| \uparrow, \downarrow ; \uparrow, \downarrow ; \Omega>_{e}, \quad \mid \phi_{k}>=\left(\hat{c}_{k, \downarrow}^{+}\left|\uparrow, \downarrow ; \uparrow, 0 ; \Omega>_{e}-\hat{c}_{k, \uparrow}^{+}\right| \uparrow, \downarrow ; 0, \downarrow ; \Omega>_{e}\right) / \sqrt{2}
\end{array}
$$

(fig. 2a). The first four indexes in the electron function $\mid \gamma_{s \uparrow}, \gamma_{s \downarrow} ; \gamma_{p \uparrow}, \gamma_{p \downarrow} ; \Omega>_{e}$ represent the occupations of the QD; $\Omega$ denotes all states of the Fermi sea with $k<k_{F}$, where $k_{F}$ is the 


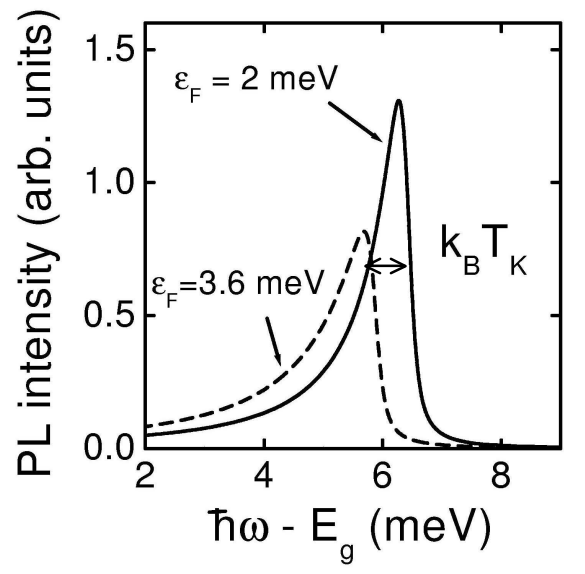

FIG. 3: PL line of $X^{2-, t}$ exciton; $U_{d o t}=64 \mathrm{meV}$ and $\Delta=1 \mathrm{meV}$.

Fermi momentum. The function $\mid \uparrow, 0>_{h}$ describes the hole with $J=+3 / 2$. Note that the electronic part of the function (44) is a singlet state with $S_{e}=0$. By using the Hamiltonian (11), we find the unknown coefficients in eq. 4 and the ground state energy $E_{i}=E_{X 2-}+\epsilon_{F}-\delta$. The lowering of energy $\delta$ plays the role of a Kondo temperature, $T_{K}$. If $E_{p}^{e}+U_{p}-\epsilon_{F}>\delta$, we obtain

$$
k_{B} T_{K}=\delta=\left(D-\epsilon_{F}\right) e^{-\frac{\pi\left(E_{p}^{e}+U_{p}-\epsilon_{F}\right)}{2 \Delta}},
$$

7]. Here $\Delta=\pi V_{0}^{2} \rho_{0}$ is the broadening and $\rho_{0}$ is the $2 \mathrm{D}$ density of states (DOS). $U_{p}$ is the Coulomb-blockade energy for the $p$-state; $D$ is the cut-off parameter for the tunnel matrix element $V_{k}: V_{k}=V_{0}$ for $\epsilon<D$ and 0 elsewhere [8]. For example, $T_{K} \sim 7-14 \mathrm{~K}$ for $\epsilon_{F}=2-3.6 \mathrm{meV}, \Delta=1 \mathrm{meV}$, and $D \sim 30 \mathrm{meV}$.

The PL spectrum can be calculated by using eqs. 2 and 4 . In the regime $\epsilon_{F}<\epsilon_{1 \rightarrow 2}$, the PL spectrum contains three lines. The main lines are singlet and triplet states of $X^{2-}$. The third structure is a shake-up line due to $X^{3-}$. The most intense, triplet line has an asymmetric shape [9]:

$$
I_{X 2-, t}=\frac{3}{4} V_{o p t}^{2} \frac{2 \Delta A_{0}^{2}}{\pi} \int_{0}^{D-\epsilon_{F}} d \epsilon \frac{1}{\left(\epsilon+k_{B} T_{K}\right)^{2}} R e \frac{-i}{\hbar \omega-\Delta E_{X 2-, t}^{\prime}+\epsilon-i \gamma_{t}},
$$

where $\Delta E_{X 2-, t}^{\prime}=\Delta E_{X 2-, t}-k_{B} T_{K}$ is the renormalized exciton energy, $\Delta E_{X 2-, t}$ is the optical energy in the absence of the $2 \mathrm{D}$ gas, and $\gamma_{t}$ describes the phonon-induced broadening of the final state. Typically, the broadening of the triplet intra-dot state $\gamma_{t}$ is small, since the energy relaxation in this case involves a spin-flip process. If $K T_{K} \gg \gamma_{t}$, the triplet line is asymmetric and its width is equal to $K T_{K}$ (fig. 3). The shape and width of the line originate from the peak of the spectral density of states (SDOS) at the Fermi level in the initial Kondo state given by eq. 4. The line shape and peak position in fig. 4 depend on the Fermi energy and, therefore, are voltage-dependent. Besides, we think that the line width is strongly temperature-dependent, because the Kondo peak in the SDOS vanishes as the temperature increases [7]. 


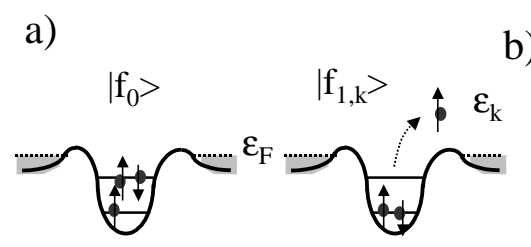

b)

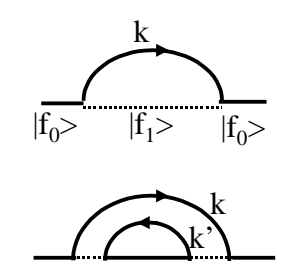

c)

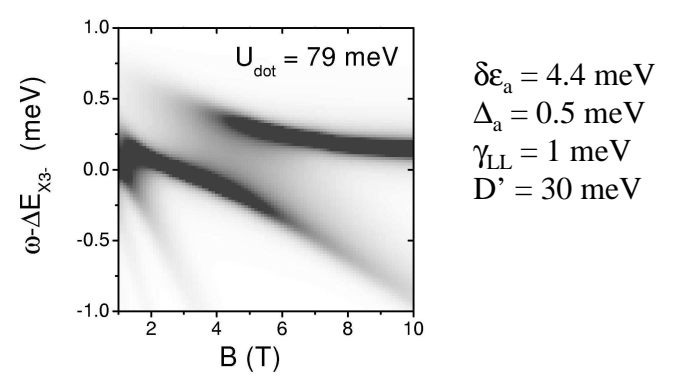

FIG. 4: (a) Final intra-dot states of the exciton $X^{3-}$. (b) Non-crossing diagrams. (c) The PL spectrum of $X^{3-}$ in the magnetic field; $\gamma_{L L}$ is the Landau-level broadening.

\section{EXCITON $X^{3-}$ WITH $S_{d o t}=0$ : AUGER PROCESSES}

In the case $\epsilon_{F}>\epsilon_{1 \rightarrow 2}$, the main peak in the spectrum comes from the exciton $X^{3-}$ where there are two completely filled electron levels. Its intra-dot electron spin is zero. Thus, the Kondo effect does not occur in the initial state. However, after photon emission, the final state of the exciton $X^{3-}$ is excited and can interact with the continuum of states due to the Auger-like process induced by the intra-dot Coulomb interaction (fig. 4a). In such a process, the final state is a linear combination of the wave functions $\left|f_{0}>=\right| \uparrow, 0 ; \uparrow, \downarrow ; \Omega>$ and $\left|f_{1, k}>=\hat{c}_{k}^{+}\right| \uparrow, \downarrow ; 0,0 ; \Omega>$, where $k>k_{F}$ [10]. The energies of the above states can be written as $\epsilon_{0}=E_{0}^{d o t}$ and $\epsilon_{1, k}=E_{1}^{d o t}+\epsilon_{k}$, where $E_{0}^{\text {dot }}$ and $E_{1}^{\text {dot }}$ are intra-dot energies and $\epsilon_{k}$ is the kinetic energy. The parameter $\delta \epsilon_{a}=E_{0}^{d o t}-E_{1}^{\text {dot }}$ determines the excess kinetic energy, when one of the QD electrons is excited into the continuum (fig. 4a).

By using non-crossing diagrams (fig. 4b), we write the Green function (2) in the form

$$
I(\omega)=V_{o p t}^{2} \operatorname{Re}\left[\frac{-i}{\tilde{\omega}-i 0-\Sigma_{1}}\right], \quad \Sigma_{1}=\int_{0}^{\infty} \frac{W_{\epsilon}^{2} \rho(\epsilon)\left[1-f_{\epsilon}\right] d \epsilon}{\tilde{\omega}-\delta \epsilon_{a}+\epsilon-i 0-\int_{0}^{\infty} \frac{\rho\left(\epsilon^{\prime}\right) W_{\epsilon^{\prime}}^{2} f_{\epsilon^{\prime}} d \epsilon^{\prime}}{\tilde{\omega}+\epsilon-\epsilon^{\prime}-i 0}},
$$

where $\Delta E_{X 3-}$ is the optical-line energy, $\tilde{\omega}=\omega-\Delta E_{X 3-}, \rho$ is the DOS and $f_{\epsilon}$ is the Fermidistribution function; the Auger matrix element $W_{\epsilon_{k}}=<f_{0}\left|U_{\text {Coul }}\right| f_{1, k}>$, where $U_{\text {Coul }}$ is the Coulomb interaction. For the parameter $W_{\epsilon}$, we assume: $W_{\epsilon}=W_{0}$ if $\epsilon<D^{\prime}$ and 0 elsewhere. In our approach, the final states in the PL process are described by the noninteracting Anderson model, in which the Auger matrix element plays the role of tunnel element $V_{k}$.

(i) Interaction with the continuum: empty wetting layer

We now assume that the QD is sufficiently deep and the wetting layer is empty; $U_{d o t}=$ $79 \mathrm{meV}$. In this case, we can put $f(\epsilon)=0$. In the absence of a magnetic field, the PL peak (eq. 7) is close to a Lorentzian. The broadening is given by $\Delta_{a}=\pi W_{0}^{2} \rho_{0}$. The estimated Auger-broadening $\Delta_{a}$ can be as high as $2 \mathrm{meV}$ [9, 11]. Note that this mechanism 
of broadening is found in experimental spectra of $X^{3-}[3]$. In the magnetic field $B$, the DOS becomes strongly modulated, due to formation of Landau levels,

$$
\rho(\epsilon)=\rho_{0} \frac{\hbar \omega_{c}}{\sqrt{\pi} \gamma_{L L}} \sum_{n} e^{-\frac{\left(\epsilon-\epsilon_{n}\right)^{2}}{\gamma_{L L}^{2}}},
$$

where $\gamma_{L L}$ is the Landau level broadening, $\epsilon_{n}=(n+1 / 2) \hbar \omega_{c}$ is the Landau level energy, and $\omega_{c}$ is the cyclotron frequency. In this case, the optical energies demonstrate anticrossings between the terms: $\tilde{\omega}=0$ and $\tilde{\omega}-\delta \epsilon_{a}+(n+1 / 2) \hbar \omega_{c}=0$ (fig. $\left.4 \mathrm{c}\right)$. The anticrossing strength increases with the magnetic field as $\sqrt{\Delta_{a} \hbar \omega_{c}}$. This is due an increase of the density of states.

(ii) Spectrum of $X^{3-}$ in the presence of a $2 d$ gas

If $T=0, B=0$ and $\delta \epsilon_{a}-\epsilon_{F}>\Delta_{a}$, the line is again close to a Lorentzian with a high-energy cut-off at the frequency $\tilde{\omega}=\delta \epsilon_{a}-\epsilon_{F}$. If $B \neq 0$, the PL spectrum in the presence of $2 \mathrm{D}$ gas is more complex due to electron-hole excitations in the Fermi sea.

\section{TRANSITION FROM $X^{2-}$ TO $X^{3-}$}

When $\epsilon_{F} \sim \epsilon_{1 \rightarrow 2}$, the ground state $X^{2-}$ changes to the state $X^{3-}$. In the transition regime, the exciton strongly couples with the Fermi sea. Hence, we can expect that the optical energies should strongly depend on $\epsilon_{F}$. In fig. $2 \mathrm{~b}$, we show this effect by using a simplified, zero bandwidth model [9, 12]. We can see that the energies $\Delta E_{X 2-, t(s)}$ decrease with $\epsilon_{F}$ and, thus, are voltage-dependent. Such a behavior has been observed in recent experiments [3].

\section{CONCLUSIONS}

Novel exciton states can appear in QDs interacting with the Fermi gas. The specific feature of these states is a "cloud" of electrons around the charged exciton. The radius of the Kondo-Anderson excitons is given by the size of the electron cloud and can be greater than the size of QD. We describe several striking manifestations of such excitons in PL

spectra. Some of described phenomena are seen in experimental spectra of InAs QDs [3].

[1] D. Goldhaber-Gordon et al., Nature (London) 391, 156 (1998); S. M. Cronenwett, T. H. Oosterkamp, and L. P. Kouwenhoven, Science 281, 540 (1998); L. I. Glazman and M. E. Raikh, Pis'ma Zh. Eksp. Teor. Fiz. 67, 1276 (1988) [JETP Lett. 47, 452 (1988)]; T. K. Ng and P. A. Lee, Phys. Rev. Lett. 61, 1768 (1988).

[2] T. V. Shahbazyan, I. E. Perakis, and M. E. Raikh, Phys. Rev. Lett. 84, 5896 (2000); K. Kikoin and Y. Avishai, Phys. Rev. B 62, 4647 (2000).

[3] R. J. Warburton et al., in the Proceedings of MRS Fall Meeting (Boston, 2002).

[4] R. J. Warburton et al, Nature (London) 405, 926 (2000).

[5] F. Findeis et al., Phys. Rev. B 63, 121309 (2001).

[6] R. J. Luyken et al., Appl. Phys. Lett. 74, 2486 (1999).

[7] A. C. Hewson, The Kondo Problem to Heavy Fermions (Cambridge University Press, Cambridge, 1993); O. Gunnarsson and K. Schonhammer, Phys. Rev. B 28, 4315 (1983). 
[8] The matrix element $V_{k}$ decreases with the kinetic energy $\epsilon_{k}$. The cut-off parameter $D$ can be estimated as $\hbar^{2} / m^{*} a^{2}$, where $a$ and $m^{*}$ are the QD lateral size and electron mass, respectively.

[9] A. O. Govorov et al., to be published; cond-mat/0211155

[10] Besides, the final state includes electron-hole pairs in the Fermi sea.

[11] R. Ferreira and G. Bastard, Appl. Phys. Lett. 74, 2818 (1999).

[12] In the zero bandwidth model, we replace the Fermi sea by a single "delocalized" state of energy $\epsilon_{F}$ [7]. 